\title{
Revisiting the theory of the evolution of pick-up ion distributions: magnetic or adiabatic cooling?
}

\author{
H. J. Fahr \\ Argelander Institut für Astronomie, Astrophysics Department, Univ. of Bonn, Auf dem Huegel 71, 53121 Bonn, Germany
}

Received: 6 September 2007 - Revised: 29 November 2007 - Accepted: 10 December 2007 - Published: 2 January 2008

\begin{abstract}
We study the phasespace behaviour of heliospheric pick-up ions after the time of their injection as newly created ions into the solar wind bulk flow from either charge exchange or photoionization of interplanetary neutral atoms. As interaction with the ambient MHD wave fields we allow for rapid pitch angle diffusion, but for the beginning of this paper we shall neglect the effect of quasilinear or nonlinear energy diffusion (Fermi-2 acceleration) induced by counterflowing ambient waves. In the up-to-now literature connected with the convection of pick-up ions by the solar wind only adiabatic cooling of these ions is considered which in the solar wind frame takes care of filling the gap between the injection energy and energies of the thermal bulk of solar wind ions. Here we reinvestigate the basics of the theory behind this assumption of adiabatic pick-up ion reactions and correlated predictions derived from it. We then compare it with the new assumption of a pure magnetic cooling of pickup ions simply resulting from their being convected in an interplanetary magnetic field which decreases in magnitude with increase of solar distance. We compare the results for pick-up ion distribution functions derived along both ways and can point out essential differences of observational and diagnostic relevance. Furthermore we then include stochastic acceleration processes by wave-particle interactions. As we can show, magnetic cooling in conjunction with diffusive acceleration by wave-particle interaction allows for an unbroken power law with the unique power index $\gamma=-5$ beginning from lowest velocities up to highest energy particles of about $100 \mathrm{KeV}$ which just marginally can be in resonance with magnetoacoustic turbulences. Consequences for the resulting pick-up ion pressures are also analysed.
\end{abstract}

Keywords. Space plasma physics (Kinetic and MHD theory; Transport processes; Wave-particle interactions)

Correspondence to: H. J. Fahr

(hfahr@astro.uni-bonn.de)

\section{Introduction}

It is common knowledge since years that suprathermal pickup ions are produced from neutral atoms all over the inner heliosphere, while their phase-space propagation is a subject much less settled in the present literature. Especially there is an ongoing debate of how efficiently pick-up ions just after the pick-up process are accelerated to higher energies due to nonlinear wave-particle interactions (see e.g. Fisk, 1976a, b; Lee, 1982; Isenberg, 1987; Bogdan et al., 1991; Chalov and Fahr, 1996; Chalov and Fahr, 1998; Fahr and Lay, 2003; Chalov et al., 2004). There is perhaps some hint given by the behaviour of the solar wind proton temperature with solar distance. Namely its observed non-adiabatic temperature behavior proves that a specific solar wind proton heating must operate in the outer heliosphere which, as meanwhile discussed in length, can only be due to energy absorption from pick-up ion generated turbulence (see Smith et al., 2001; Chashei and Fahr, 2003; Chashei et al., 2003). The ionization of interstellar $\mathrm{H}$-atoms penetrating the heliosphere results in the formation of keV-energetic protons in the supersonic solar wind regime which may be called primary pick-up ions (PUI's*). The velocity distribution of these newly produced PUI's* is a toroidal function which is highly anisotropic and unstable. With the free energy of this unstable distribution these PUI's* drive Alfvenic turbulence which by itself selfconsistently enforces pitchangle isotropization of the initial velocity distribution and energy diffusion to occur (see Chalov et al., 2004, 2006).

Due to wave-wave coupling the wave energy generated by PUI's* at the injection wavelength $\lambda_{i}=U / \Omega_{p}(U=$ solar wind speed; $\Omega_{p}=$ local ion gyrofrequency) is then transported in wavevector space both to smaller wavelengths where it can be absorbed by solar wind protons and to larger wavelengths where it is reabsorbed by PUI's. This effect is seen as the main reason for solar wind proton heating occuring in the outer heliosphere (Smith et al., 2001; Fahr and

Published by Copernicus Publications on behalf of the European Geosciences Union. 
Chashei, 2002; Chashei et al., 2003). From estimations it is, however, evident that only a small fraction of about 5 percent of the PUI-generated wave energy reappears in the observed proton temperature profiles, raising the question where the major portion of the wave energy produced during the primary pick-up process is going.

A thermodynamic study of the solar wind proton/PUI twofluid temperature behaviour at larger solar distances under consistent wave-particle energy sharing between protons and PUI's was carried out (Chashei et al., 2003). This study revealed the non-adiabatic proton temperature behaviour as well as a nearly isothermal pick-up ion behaviour. To clarify more quantitatively the energy branching kinetic and spectral details of the relevant transfer processes had to be investigated. A detailed numerical study of the PUI velocity distribution and the spectral Alfvénic wave power evolution has meanwhile been carried out (Chalov et al., 2004, 2006a, b) and consisted in the simultaneous solution of a coupled system of equations consistently describing both the isotropic velocity distribution function of PUI's and the spectral wave power intensity.

As one can see from this study the largest portion of the self-generated wave energy is reabsorbed by PUI's themselves as a result of the cyclotron resonant interaction and leads to PUI-acceleration. This just results in the energization of pick-up protons due to the stochastic acceleration process probably finally producing ubiquitous power-law PUItails extended to energies much higher than the PUI injection energy (i.e. about $1 \mathrm{KeV}$ ). These tails are seen everywhere in the solar system (see Fisk et al., 2000; Fisk and Gloeckler, 2006,2007 ) and they essentially reduce the amount of the wave energy which is left for absorption by solar wind protons. In the following paper we shall, however, not primarily look into details of the diffusive acceleration of PUI's and the terms describing this phasespace propagation process, but we shall reconsider those terms in the phasespace transport equation describing processes that are effective in the redistribution of PUI energies from the injection energy threshold to lower energies by adiabatic ion reactions to changing magnetic fields in the plasma box comoving with the solar wind.

\section{Kinetics of magnetic cooling}

In a magnetic field $B$, variable in magnitude with solar distance $r$, freely wind-convected ions have to conserve their magnetic moment $M=m v_{\perp}^{2} / 2 B$, where $m, v_{\perp}, B$ denote the ion mass, the component of the ion velocity $v$ perpendicular to the magnetic field, and the interplanetary magnetic field, respectively. The gyro-averaged Lorentz force acts on these ions by decreasing $v_{\perp}^{2}$ at the decrease of $B$. For an isotropic distribution function this can be interpreted as an induced convection in velocity space due to a force connected with a temporal velocity change $\dot{v}_{m}=\sqrt{\frac{2}{3}}\left\langle\dot{v}_{\perp}\right\rangle$ (see Fahr and Lay, 2000).
The representative Boltzmann-Vlasow equation (BVE) in the "solar" rest frame (SF) under these conditions for the stationary case is given by:

$$
\begin{aligned}
\left(\boldsymbol{U} \cdot \nabla_{r}\right) f+\left(\frac{d}{d t} \boldsymbol{v}_{m} \cdot \nabla_{v}\right) f & =U \frac{\partial f}{\partial r}+U \frac{\partial v_{m}}{\partial r} \frac{\partial f}{\partial v} \\
& =P(r, v)
\end{aligned}
$$

while the corresponding Boltzmann-Vlasow equation (BVE) in the "solar wind" rest frame (WF) has the following form:

$$
\frac{\partial \tilde{f}}{\partial t}+\frac{1}{v^{2}} \frac{\partial}{\partial v}\left(v^{2} \dot{v}_{m} \tilde{f}\right)=\tilde{P}(t, v)
$$

where the second term on the left hand side describes the velocity-space divergence of the phasespace flow connected with the acceleration $\dot{v}_{m}=d v_{m} / d t$. The coordinate $t=t(r)$ denotes the proper time in the co-moving reference frame (WF). Furthermore $\tilde{P}(r, v)$ is the local ion injection rate given by $\tilde{P}(r, v)=\beta(r) \frac{1}{4 \pi v^{2}} \delta(v-U)$ with $\beta(r)$ being the local pick-up ion production rate. In contrast, in the SF, taking $\mathrm{H}$-atom velocities as negligibly small with respect to the wind velocity $U$, the injection term is given by $P(r, v)=\beta(r) \frac{1}{4 \pi \cdot v^{2}} \delta(v)$.

Furthermore $\dot{v}_{m}$ denotes the magnetic velocity decrease of particles with a velocity $v$, when they are convected out with the solar wind bulk flow at a mean velocity $U$ to larger distances where the co-convected interplanetary magnetic field $B$ is decreased. If in fact the field decreases by $(1 / r)$, as in case of the nearly azimuthal, distant Parker field, then, with the pitchangle average $\left\langle v_{\perp}\right\rangle_{\vartheta}$ of the velocity component $v_{\perp}$ for pitchangle isotropic distribution functions, i.e. with $\left\langle v_{\perp}^{2}\right\rangle_{\vartheta}=\frac{2}{3} v^{2}$, one finds the following $v$ - dependent magnetic velocity-space drift

$\dot{v}_{m}=-U \frac{v}{r}$

and as well its associated radial gradient

$\frac{\partial v_{m}}{\partial r}=\frac{1}{U} \dot{v}_{m}=-\frac{v}{r}$

As a reminder one may note, that for the more radial field at smaller distances, falling off with $r^{-2}$, one simply gets an additional factor 2 in the above relations.

If no rapid pitchangle isotropisation can be assumed, magnetic cooling as derived above should evidently only cool down the velocity degree of freedom perpendicular to the magnetic field and thus should lead to increasingly anisotropic distribution functions. This problem, especially coming up in the radially expanding solar wind plasma, was already clearly seen by Griffel and Davies (1969) and reviewed by Fahr and Shizgal (1983). These authors claimed for effective collision frequencies to act in the solar wind region inside $1 \mathrm{AU}$, which should be much higher than the actual Coulomb collision frequencies, in order to keep the ion and electron velocity distributions sufficiently isotropic, as 
is seen in observations. Interestingly enough, one can, however, show that the problem, seen up from these early days, is essentially solved by additionally considering the second CGL invariant, $C G L_{2}=\left(P_{\|} B^{2} / \rho^{3}\right)$, in addition to the first CGL-invariant, $C G L_{1}=\left(P_{\perp} / B \rho\right)$. Here $P_{\perp, \|}$ and $\rho$ denote the components of the ion pressure and the solar wind ion density, respectively. Conservation of $C G L_{2}$ namely implies an independent cooling of the velocity degree parallel to the magnetic field, i.e. of $v_{\|}$(see Siewert and Fahr, 2007).

For larger distances $r \geq r_{0}=5 \mathrm{AU}$ with magnetic fields decreasing like $(1 / r)$ this is quantitatively seen in the following way:

$$
\begin{aligned}
C G L_{2}=\left(P_{\|} B^{2} / \rho^{3}\right) & =\frac{\frac{1}{2} \rho\left\langle v_{\|}^{2}\right\rangle B^{2}}{\rho^{3}}=\frac{1}{2}\left\langle v_{\|}^{2}\right\rangle\left(\frac{B}{\rho}\right)^{2} \\
& =\frac{1}{2}\left(\frac{B_{0}}{\rho_{0} r_{0}}\right)^{2}\left\langle v_{\|}^{2}\right\rangle r^{2}
\end{aligned}
$$

As we have shown in Siewert and Fahr (2007) the above relation is fulfilled, if

$v_{\|}^{2} r^{2}=$ const $=C_{2}$

is valid for all individual ions. Then this above relation simply requires that

$\frac{d v_{\|}}{d r}=-\frac{2 C_{2}}{v_{\|} r^{3}}=-\frac{v_{\|}}{r}$

This interestingly enough demonstrates that conservation of the first and second CGL invariants requires that both velocity degrees of freedom, i.e. $v_{\|}$and $v_{\perp}$, cool by the same rate at the expansion of the solar wind, thus at least at larger distances tending to keep pitchangle isotropic distribution functions.

Ions which are picked up at $r_{v}$ with a velocity $U$ will, if nothing else happens, have adiabatically, or better say "magnetically", cooled down to a velocity $v$ at $r$, if the following relation is fulfilled:

$r_{v}(v)=\frac{v}{U} r$

Thus the injection of freshly created pick-up ions at $r_{v}(v)$ with an initial velocity $v=U$ will be responsible for ions with velocity $v$ at $r$.

Taking all these constraints together one finally finds, when reminding that the time and distance coordinates are related to eachother by $d r=U d t$, that the solution for $\tilde{f}$ in the WF can be given by:

$\tilde{f}=\frac{1}{2 \pi} \frac{r \beta\left(\frac{v}{U} r\right)}{U} v^{-3}$

The fact that the above distribution function $\tilde{f}$ actually solves the BVE given by Eq. (2) is explicitly proven in the Appendix $\mathrm{A}$ of this paper.
The above distribution function can now be further developed for larger solar distances $r \geq r_{0}=5 \mathrm{AU}$ in the upwind hemisphere. At large enough solar distances the upwind $\mathrm{H}$ atom density can be considered as essentially constant allowing to assume that $n_{H}\left(\frac{v}{U} r\right)=n_{H}(r)=n_{H, \infty}$, which is an acceptable approximation for solar distances $r \geq 5 \mathrm{AU}$ and velocities $v \geq 0.2 U$. Then one evidently finds

$$
\begin{aligned}
f & =\frac{r}{2 \pi U} v_{e x, E} r_{E}^{2}\left(\frac{v}{U} r\right)^{-2} n_{H, \infty} v^{-3} \\
& =\frac{v_{e x, E} r_{E}^{2} U}{2 \pi r} n_{H, \infty} v^{-5}
\end{aligned}
$$

The astonishing fact that one should recognize in this function above is that, under pure magnetic cooling, the resulting PUI distribution function is a power law with the interesting power index $\alpha=-5$, a power index which, astonishingly enough, was also found by Fisk and Gloeckler (2006, 2007), however, as result for the quasi-equilibrium state established between magnetoacoustically driven ion energy diffusion and magnetoacoustic turbulence generation. This seems to open up the interesting possibility that the PUI distribution could perhaps be characterized by a thorough unbroken power law with the transitive uniform power index " -5 " running from regions of velocities where energy diffusion by wave-particle interaction operates (Fisk and Gloeckler, 2007) down to the region where this process stops to operate due to non-existence of resonant conditions with the magnetoacoustic wave fields.

\section{Adiabatic cooling as a contrast}

As a competing approach to the above presented derivation in the up-to-now literature a phasespace transport equation has always been applied which in its general form is borrowed from the CR-transport equation originally developed for cosmic rays (see Parker, 1965; Gleeson and Axford, 1967). This equation, though well approved for the high energy range of cosmic rays, may, however, become questionable, if it is to be applied to low energy particles like pick-up ions. This we want to demonstrate in the following.

The argumentation for the adiabatic cooling term that appears in the CR transport equation (Parker, 1965; Jokipii, 1971) and also is used in the PUI transport equation (see e.g. Isenberg, 1987; Chalov et al., 1995, 1997; Mall, 2000) usually runs as follows: If the pressure of pick-up ions does work at the volume expansion, connected with the expansion of a spherically diverging solar wind flow, then thermodynamically a loss of internal pick-up ion energy $\Xi$ (i.e. enthalpy) in the comoving frame is to be expected to occur. This then results in the following thermodynamic relation

$\frac{d \Xi}{d t}=n V \frac{d \chi}{d t}=-P \frac{d V}{d t}$

where $\chi=\Xi / n V$ here denotes the enthalpy per particle. This effect is expected from thermodynamic principals under 
conditions where adiabatic reactions of the gas in the expanding flow can be expected, i.e. under subsonic expansion rates and a quasi-isentropic gas behaviour.

The latter two conditions may, however, not be fulfilled in the region of supersonic solar wind expansion with no rapid relaxation processes like collisions or wave-particle interactions being involved (N.B.: A piston gas with a piston expanding supersonically!). That means at larger solar distances, i.e. beyond $1 \mathrm{AU}$, conditions for low energy particles are in contradiction to the assumption of the validity of the above mentioned thermodynamic relation.

This is, of course, different for high energy particles of a quasi-massless species " $i$ " with velocities $v_{i}$ or sound velocities $c_{i}=\sqrt{d P_{i} / d \rho_{i}}$ much greater than the solar wind velocity. These latter particles, like galactic or anomalous cosmic rays, undergoing scattering processes at stochastically distributed, magnetic inhomogeneities, quasi-frozen into the supersonically expanding solar wind, will in fact behave nearly isentropic and adiabatic and may approximately fullfill the above thermodynamic relation (e.g. see Toptygin, 1985). This is not so, however, for low energy subsonic ions. As both exospheric solar wind theories (see Lemaire and Scherer, 1971, Marsch and Livi, 1985) and in-situ plasma observations (Marsch et al., 1981) can clearly show, solar wind ions at their expansion evidently behave non-adiabatic and nonisentropic in the supersonic solar wind.

Nevertheless, since the isentropy assumption and the above thermodynamic relation has often been used also for low energy ions in the literature of the past (see e.g. Vasyliunas and Siscoe, 1976; Isenberg, 1987; Chalov and Fahr, 1995, 1998; Mall, 2000) we shall look into this approach here again and want to compare it with the approach made above for pure magnetic cooling.

For a spherically symmetric radial solar wind with constant bulk velocity $U$ the change of the comoving proper volume with time is given by

$\frac{d V}{d t}=(\nabla \cdot \boldsymbol{U}) V=2 \frac{U}{r} V$

and thus with the relation further above delivers the following equation

$n \frac{d \chi}{d t}=-P \frac{1}{V} \frac{d V}{d t}=-2 P \frac{U}{r}$

One now can play a trick and replace without a good physical basis macroscopic thermodynamic by corresponding kinetic quantities, taking the enthalpy per particle and the pressure in the form

$\chi=\frac{\gamma}{\gamma-1}\left\langle\frac{p^{2}}{2 m}\right\rangle$

and

$P=n k T \simeq n \frac{2}{3}\left\langle\frac{p^{2}}{2 m}\right\rangle$ which then leads one to the relation

$n \frac{1}{P} \frac{d \chi}{d t} \simeq \frac{3}{2} \frac{\gamma}{\gamma-1} \frac{\frac{d}{d t}\left\langle p^{2}\right\rangle}{\left\langle p^{2}\right\rangle}=-2 \frac{U}{r}$

Transcribing now this macroscopic relation to properties of individual particles, not caring for probability weights only defined by the distribution function, - in general a highly problematic and questionable procedure -, will then finally yield

$\frac{3}{2} \frac{\gamma}{\gamma-1} \frac{2 p \frac{d}{d t} p}{p^{2}}=-2 \frac{U}{r}$

which evidently leads to the resulting so-called adiabatic momentum change given by

$\left(\frac{d p}{d t}\right)_{a d}=-\frac{2}{3} \frac{\gamma-1}{\gamma} \frac{U}{r} p$

This strongly simplified expression has been introduced as well into the CR transport equation as also into the pick-up ion Boltzmann equation by taking $\gamma \gg 1$ and understanding $\left(\frac{d p}{d t}\right)_{a d}$ as an "adiabatic force" acting on the comoving particles. For PUI's this then leads to the following BVE

$U \frac{\partial f}{\partial r}-\frac{2}{3} U \frac{v}{r} \frac{\partial f}{\partial v}=\beta(r) \frac{1}{2 v^{2}} \delta(v-U)$

It has first been shown by Vasyliunas and Siscoe (1976) that this differential equation is solved by

$f_{\text {pui }}(r, v)=\frac{3 r}{8 \pi U^{4}} \beta\left(r_{v, a d}\right) \cdot\left(\frac{v}{U}\right)^{3 / 2}$

where $r_{v, a d}$ denotes that specific place, conjugated to $r$ under adiabatic deceleration due to the adiabatic velocity drift, which is given by

$r_{v, a d}=r \cdot\left(\frac{v}{U}\right)^{3 / 2}$

The above expression then translates into the following form of the distribution function when $\beta\left(r_{v, a d}\right)$ is expressed with the charge exchange ionization frequency:

$f_{\text {pui }}(r, v)=\frac{3 v_{e x, E} r_{E}^{2}}{8 \pi r U^{4}} n_{H}\left(r_{v, a d}\right) \cdot\left(\frac{v}{U}\right)^{-3 / 2}$

which for larger distances when again setting $n_{H}\left(r_{v, a d}\right) \simeq n_{H}(r) \simeq n_{H, \infty}$ needs then to be compared with the expression found for purely magnetic cooling (see Eq. 10) which latter when given for the normalized velocity argument $(v / U)$ takes the form:

$f_{\text {pui }}(r, v)=\frac{v_{e x, E} r_{E}^{2}}{2 \pi r U^{4}} n_{H, \infty}\left(\frac{v}{U}\right)^{-5}$

Both functions have identical $r$-dependences and both are power law distributions, however, the main difference between these two distribution functions, as can clearly be recognized, is the difference in the prevailing power law indices, 
i.e. " $-3 / 2$ " for the adiabatic cooling instead of " -5 " for the magnetic cooling. This also implies interestingly enough that under quasi-equilibrium energy diffusion driven by magnetoacoustic turbulences (see Fisk and Gloeckler, 2007) together with adiabatic cooling a broken power-law distribution would result, similar to the one presented already by Isenberg (1987).

\section{Distribution and pressure of wave-accelerated pick- up ions}

As we have shown before pure magnetic cooling of pick-up ions leads to a power law distribution with the power index $\gamma_{m}=-5$. However, from the theory behind this power law one can conclude that it should only be governing the PUI distribution from the injection threshold downward, i.e. it should be valid for PUI velocities of $v \leq U$. The question thus arises how the PUI distribution might look in regions $v \geq U$. In this region pick-up ions have definitely been observed (Gloeckler et al., 1993; Geiss et al., 1994; Möbius et al., 1996, 1998) and thus they must have been transported there by acceleration processes. The idea is that this acceleration takes place by means of diffusive Fermi-2 acceleration via quasilinear interaction of ions with either Alfvenic or magnetoacoustic turbulences (see Isenberg, 1987; Chalov and Fahr, 1998, 2000; Toptygin, 1985). In our calculations of the ongoing paper here we shall rely on the well-based argument given by Fisk and Gloeckler $(2006,2007)$ that pickup ions under resonant interaction with ambient magnetoacoustic turbulences tend to develope in the range of resonant ions a saturated, unbroken power-law distribution just with a unique spectral power index of $\gamma_{s}=-5$.

Adopting this as a fact, well supported both by theory and observations, we now calculate quantitatively the absolute spectral intensity of this power-law distribution from the physical principles behind. We begin with a PUI-distribution function given in the form:

$$
f_{\text {pui }}(r, v)=f_{\text {pui }, 0} \cdot\left(v / v_{0}\right)^{\gamma_{s}}
$$

which yields the PUI density in the form:

$$
n_{\text {pui }}(r)=4 \pi f_{\text {pui }, 0}(r) \cdot \int_{v_{0}}^{v_{\infty}}\left(w / w_{0}\right)^{-5} w^{2} d w
$$

where $f_{\text {pui, } 0}(r)$ is a local normalization value, and $v_{0}$ and $v_{\infty}$ are lower and upper velocity resonance limits of the PUI power law. These quantities have to be fixed by physical constraints. From the above expression one first obtains the PUI density, with $x=w / w_{0}$ and with the assumption $x_{\infty}=v_{\infty} / v_{0} \gg 1$, by

$$
\begin{aligned}
n_{\text {pui }}(r) & =4 \pi f_{\text {pui }, 0}(r) \cdot v_{0}^{3} \int_{1}^{x_{\infty}} x^{-3} d x \\
& \simeq 2 \pi f_{\text {pui }, 0}(r) \cdot v_{0}^{3}
\end{aligned}
$$

and the PUI pressure as formulated by

$$
\begin{aligned}
P_{\text {pui }}(r) & =4 \pi f_{\text {pui }, 0}(r) \cdot \frac{m}{2} v_{0}^{5} \int_{1}^{x_{\infty}}(x)^{-5} x^{4} d x \\
& =4 \pi f_{\text {pui }, 0} \cdot \frac{m}{2} v_{0}^{5} \ln \left(x_{\infty}\right)
\end{aligned}
$$

As one can see, the definition of the absolute value of the PUI pressure requires the determination of the values, $f_{\text {pui, } 0 \text {, }}$ $v_{0}$ and $v_{\infty}$, which we aim at now.

To impede PUI's at some inner velocity border from completely migrating to lower energies by magnetic cooling (i.e. energy loss due to conservation of the magnetic moment at convection to regions with smaller magnetic fields!) it should be guaranteed that they are restored with the adequate rate just at this lower border $v=v_{0}$ of the power-law distribution, where energy diffusion stops to operate due to loss of resonance conditions with the turbulence. This means that near the pick-up velocity border, the particle loss rate due to magnetic cooling must be compensated by a diffusive flow due to the established energy diffusion rate. This means that the differential particle fluxes in velocity space should just be identical, i.e. $(d j)_{m}=(d j)_{\text {diff }}$.

Hereby the differential flux in velocity space due to magnetic ion cooling at the inner velocity resonance border $v=v_{0}$ is given by

$$
\begin{aligned}
(d j)_{m} & =4 \pi v_{0}^{2} f_{\text {pui }}\left(v_{0}\right)\left(\frac{\delta v_{0}}{\delta t}\right)_{m} \\
& =4 \pi v_{0}^{2} f_{\text {pui }}\left(v_{0}\right) U\left(\frac{\delta v_{0}}{\delta r}\right)_{m}
\end{aligned}
$$

When $B$ denotes the interplanetary magnetic field which at larger distances in the ecliptic according to the Archimedian Parker spiral configuration can be assumed to be purely azimuthal and given by $B=B_{0}\left(r / r_{0}\right)^{-1}$, then yields, with $v_{\perp}^{2} / B=v^{2} \sin ^{2} \vartheta / B=$ const and the assumption of a

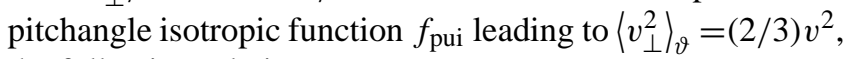
the following relation

$$
\left(\frac{\delta v_{0}}{\delta r}\right)_{m}=-\frac{1}{2} \frac{v_{0}}{r}
$$

and thus the following expression

$$
\left.(d j)_{m}=-2 \pi v_{0}^{2} f_{\text {pui }}\left(v_{0}\right) U \frac{v_{0}}{r}\right)
$$

To keep the inner border stationary, this adiabatic loss rate needs to be compensated by the energy-diffusion gain rate given by

$$
(d j)_{\text {diff }}=4 \pi v^{2} D_{v v} \frac{\partial}{\partial v} f_{\text {pui }}
$$

where $D_{v v}$ is the energy-diffusion coefficient due to interaction with compressive magnetosonic turbulences which according to Chalov et al. (2003) is given in the form:

$$
D_{v v}=D_{v v, 0} \cdot\left(\frac{U^{3}}{r_{E}}\right)\left(\frac{v}{U}\right)\left(\frac{r_{E}}{r}\right)^{3 / 4}
$$


where $D_{v v, 0}$ is a reference value at $r=r_{E}=1 \mathrm{AU}$.

$(d j)_{d i f f}=4 \pi D_{v v, 0} \cdot\left(\frac{U^{2}}{r_{E}}\right)\left(\frac{r_{E}}{r}\right)^{3 / 4} v^{3} \frac{\partial}{\partial v} f_{\text {pui }}$

yielding

$(d j)_{d i f f}=-4 \pi D_{v v, 0} \cdot\left(\frac{U^{2}}{r_{E}}\right)\left(\frac{r_{E}}{r}\right)^{3 / 4} v^{3} \frac{5}{v} f_{\text {pui }}$

evaluating at the lower velocity boundary to

$(d j)_{d i f f, 0}=-20 \pi D_{v v, 0} \cdot\left(\frac{U^{2}}{r_{E}}\right)\left(\frac{r_{E}}{r}\right)^{3 / 4} v_{0}^{2} f_{\text {pui }, 0}$

Equating adiabatic loss rate and energy diffusion rate at $v=v_{0}$ then leads to

$$
\begin{array}{r}
-2 \pi v_{0}^{2} f_{\text {pui }}\left(v_{0}\right) U \frac{v_{0}}{r}= \\
-20 \pi D_{v v, 0} \cdot\left(\frac{U^{2}}{r_{E}}\right)\left(\frac{r_{E}}{r}\right)^{3 / 4} v_{0}^{2} f_{\text {pui }, 0}
\end{array}
$$

and thus requires that the inner border is at

$$
v_{0}=5 D_{v v, 0} \cdot U\left(\frac{r}{r_{E}}\right)^{1 / 4}
$$

where the reference value for the diffusion coefficient according to Chalov et al. (2003) is given by

$D_{v v, 0}=\frac{\sqrt{\left\langle\delta U_{m}^{2}\right\rangle_{E}}}{U} \frac{r_{E}}{9 L_{m}}$

where $\left\langle\delta U_{m}^{2}\right\rangle_{E}$ and $L_{m}$ denote the wavenumber-average of the magnetosonic fluctuation power at $1 \mathrm{AU}$ and the magnetosonic correlation length. Taking values as those favoured by Chalov et al. (2003), i.e. $L_{m}=3 \mathrm{AU}$ and $\sqrt{\left\langle\delta U_{m}^{2}\right\rangle_{E}} / U=0.5$, one then finally obtains for the lower velocity boundary the mildly distance-dependent value

$v_{0}=5 \frac{\sqrt{\left\langle\delta U_{m}^{2}\right\rangle_{E}}}{U} \frac{r_{E}}{9 L_{m}} \cdot U\left(\frac{r}{r_{E}}\right)^{1 / 4} \simeq 0.2 U\left(\frac{r}{r_{E}}\right)^{1 / 4}$

and therewith one also finds using Eq. (26)

$$
\begin{aligned}
f_{\text {pui }, 0}(r) & =n_{\text {pui }}(r) \cdot \frac{1}{2 \pi v_{0}^{3}(r)} \\
& =\frac{5^{3}}{2 \pi U^{3}} n_{\text {pui }}(r) \cdot\left(\frac{r}{r_{E}}\right)^{-3 / 4}
\end{aligned}
$$

The local PUI density $n_{\text {pui }}(r)$ can be derived from the Hatom density $n_{H}(r, \theta)$ and the [charge exchange + photoionization] rate

$$
\beta_{\text {pui }}=n_{H}(r, \theta)\left[v_{\text {pui }}\right]=n_{H}(r, \theta)\left[n_{s}(r) \sigma_{e x} U+v_{i}\right]
$$

Here $n_{s}(r), v_{\text {pui }}, \sigma_{e x}, U_{s}, v_{i}$ denote solar wind proton density, the total $\mathrm{H}$-atom ionization frequency, the charge exchange cross section, the solar wind bulk velocity and the photoionization frequency. The cold-model $\mathrm{H}$-atom density, which is good enough for our purposes here, is given for the upwind hemisphere by (see Fahr, 1971)

$n_{H}(r, \theta)=n_{H, \infty} \exp \left[-\frac{\nu_{\text {pui, },} r_{0}^{2} \theta}{U r \sin \theta}\right]$

and thus in view of small $\theta$ - gradients and quasi-radial PUI flow with the accordingly simplified PUI continuity equation

$\frac{1}{r^{2}} \frac{d}{d r}\left(r^{2} U n_{\text {pui }}\right)=\beta_{\text {pui }}(r, \theta)$

leads to the following PUI density

$n_{\text {pui }}(r, \theta)=\left(\frac{r_{0}}{r}\right)^{2} n_{\text {pui }, 0}+\frac{1}{r^{2} U} \int_{r_{0}}^{r} v_{\text {pui }}(r, \theta) r^{2} d r$

\section{The upper velocity resonance border}

For the quantitative calculation of the higher PUI moments, like the PUI pressure, one more quantity needs to be defined, namely the upper velocity boundary $v_{\infty}$. We shall determine this quantity by asking for the condition which must be fulfilled for energy-diffusion to be operative at all. The important restriction to energy diffusion by nonlinear interaction with magnetosonic compressive fluctuations is that the typical diffusion periods for ions should be much larger than the associated convection periods over coherent fluctuation structures with coherence lengths $L_{m}$ that are given by $\tau_{\text {con }} \simeq L_{m} / U$. This leads to the requirement (see Chalov et al., 2003)

$L_{m} \gg \frac{v \lambda_{\|}}{3 U}$

This requirement can be taken as limiting the uppermost velocity and hence means that the largest particle velocity allowed from this requirement is

$v_{\infty} \leq \frac{3 U L_{m}}{\lambda_{\|}}$

where $\lambda_{\|}$is the mean free path for particles moving parallel to the magnetic field and given by

$\lambda_{\|}=\frac{3 v}{8} \int_{-1}^{+1} \frac{\left(1-\mu^{2}\right)^{2} d \mu}{D_{\mu \mu}}$

Here $D_{\mu \mu}$ denotes the pitchangle diffusion coefficient ( $\mu=\cos \vartheta$ denoting the pitchangle cosine) which is represented by (see Schlickeiser, 1989; or Chalov and Fahr, 1998)

$D_{\mu \mu} \sim D_{v v, 0} V_{A}^{-2}\left(\frac{U^{3}}{r_{E}}\right)\left(\frac{v}{U}\right)\left(\frac{r_{E}}{r}\right)^{3 / 4}$ 
with $V_{A}$ being the Alfvén velocity which due to $V_{A} \sim B / \sqrt{\rho}$ at distances $r \geq r_{0}$ with $B \sim r^{-1}$ and $\rho \sim r^{-2}$ can be taken as constant. This clearly shows that $\lambda_{\|}$is independent of the particle velocity and thus $v_{\infty}$ is simply given by

$v_{\infty} \leq \frac{3 U L_{m}}{\lambda_{\|_{E}}}\left(\frac{r_{E}}{r}\right)^{3 / 4}$

and reminding that the reference value of $\lambda_{\|}$at $r=r_{E}$ has been found with $\lambda_{\|_{E}} \simeq 0.3 \mathrm{AU}$ (see Chalov and Fahr, 1999) then leads to the result

$v_{\infty} \leq \frac{3 L_{m}}{\lambda_{\|_{E}}}\left(\frac{r_{E}}{r}\right)^{3 / 4} U \simeq 30\left(\frac{r_{E}}{r}\right)^{3 / 4} U$

This states that $v_{\infty}$ slowly falls off with solar distance and e.g. at $r=r_{0}=5 \mathrm{AU}$ is given by $v_{\infty} \simeq 9.6 U_{s}$. Please note that the above relation can only be applied in the supersonic solar wind region and does not include the region downstream of the termination shock.

\section{The power-law PUI pressure}

On the basis of the above derived results one then obtains the PUI pressure as given by

$$
\begin{aligned}
P_{\text {pui }}(r) & =2 \pi m \cdot f_{\text {pui }, 0} v_{0}^{5} \ln \left(x_{\infty}\right) \\
& =m \frac{\ln \left(x_{\infty}\right)}{18^{2}} U^{2}\left(\frac{r}{r_{E}}\right)^{1 / 2} n_{\text {pui }}(r)
\end{aligned}
$$

where $x_{\infty}$ for general solar wind conditions is given by

$$
\begin{aligned}
x_{\infty}=\frac{v_{\infty}}{0.2 U\left(\frac{r}{r_{E}}\right)^{1 / 4}} & =\frac{\Gamma\left(\frac{r_{E}}{r}\right)^{3 / 4} U}{0.2 U\left(\frac{r}{r_{E}}\right)^{1 / 4}} \\
& =5 \Gamma \cdot\left(\frac{r_{E}}{r}\right)
\end{aligned}
$$

where we have introduced the quantity $\Gamma=L_{m} / \lambda_{\|}$and hence leads to

$P_{\text {pui }}(r)=\frac{\ln \left(5 \Gamma \frac{r_{E}}{r}\right)}{5^{2}}\left(\frac{r}{r_{E}}\right)^{1 / 2} n_{\text {pui }}(r) m U^{2}$

At a solar distance of $r=r_{0}=5 \mathrm{AU}$ whith $\Gamma=30$ one thus finds e.g.

$$
\begin{aligned}
P_{\text {pui }}\left(r_{0}\right) & =\frac{\ln (30)}{5^{2}}(5)^{1 / 2} n_{\text {pui }}(r) m U^{2} \\
& =0.306 \cdot\left[n_{\text {pui }}(r) m U^{2}\right]
\end{aligned}
$$

and at larger distances like $50 \leq r / r_{E} \leq 80$ from the above expressions (53) and (54) one derives a ratio $\alpha_{\text {pui }}(r)$ of the local PUI pressure $P_{\text {pui }}(r)$ and the accumulated kinetic energy of freshly injected PUI's, i.e. $\left[n_{\text {pui }}(r) m U_{s}^{2}\right]$, which is only mildly variable with distance and roughly given by

$\alpha_{\text {pui }}(r)=\frac{P_{\text {pui }}(r)}{\left[n_{\text {pui }}(r) m U^{2}\right]} \simeq 0.306$

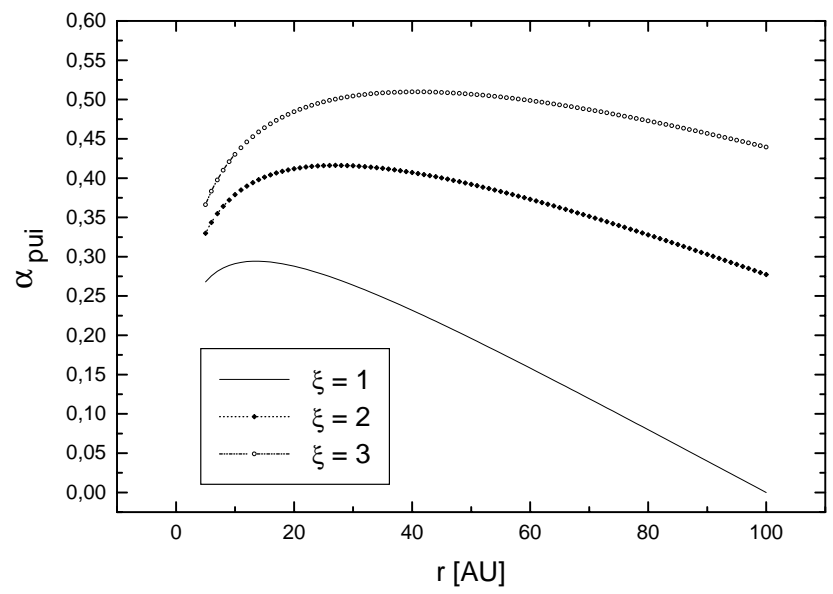

Fig. 1. Shown is the function $\alpha_{\text {pui }}$ as function of the solar distance in the upwind direction for differerent values $\Gamma=L_{m} / \lambda_{\|}=20(\xi=1)$, $30(\xi=2), 40(\xi=3)$.

When furtheron representing the PUI pressure by a PUI temperature writing $P_{\text {pui }}(r)=(3 / 2) n_{\text {pui }} K T_{\text {pui }}$, this then shows that pick-up ions at their convection towards larger distances nearly behave like an isothermal fluid with

$T_{\text {pui }}(r) \simeq 0.204 \cdot \frac{m}{K} U^{2}$

a result which was also found earlier from a different approach by Fahr (2002a, b).

To illustrate the more exact variation of $\alpha_{\text {pui }}(r)$ with solar distance $r$ as it results for different solar wind conditions during the solar activity cycle, i.e. with a typical range $20 \leq \Gamma=L_{m} / \lambda_{\|} \leq 60$, we have shown the plots given in Fig. 1 . It has perhaps to be mentioned here that $\Gamma$ essentially is variable within the solar activity cycle because of the variation of $L_{m}$, while the pitchangle scattering mean free path $\lambda_{\|}$dependent on the Alfvenic turbulence level may essentially be constant, i.e. $0.3 \leq \lambda_{\|} \leq 0.9$ (see Chalov and Fahr, 1995). The coherence scale $L_{m}$ is essentially given by the typical distance between two consecutive high velocity humps in the solar wind velocity and, according to Richardson et al. (2001), amounts to about $3 \mathrm{AU} \leq L_{m} \leq 9 \mathrm{AU}$.

\section{Solar wind deceleration by PUI pressure}

In order to compare our above result for power law PUI pressures with earlier work (Fahr and Fichtner, 1995; Fahr and Rucinski, 1999; Fahr and Scherer, 2004) we can represent the above result in the following form

$$
P_{\text {pui }}(r)=\alpha_{\text {pui }}(r) \rho_{\text {pui }}(r) U^{2}
$$

where the function $\alpha_{\text {pui }}(x)$ for $x=r / r_{E}$ is defined by

$\alpha_{\text {pui }}(x)=\frac{\ln (5 \Gamma / x)}{5^{2}}(x)^{1 / 2}$ 


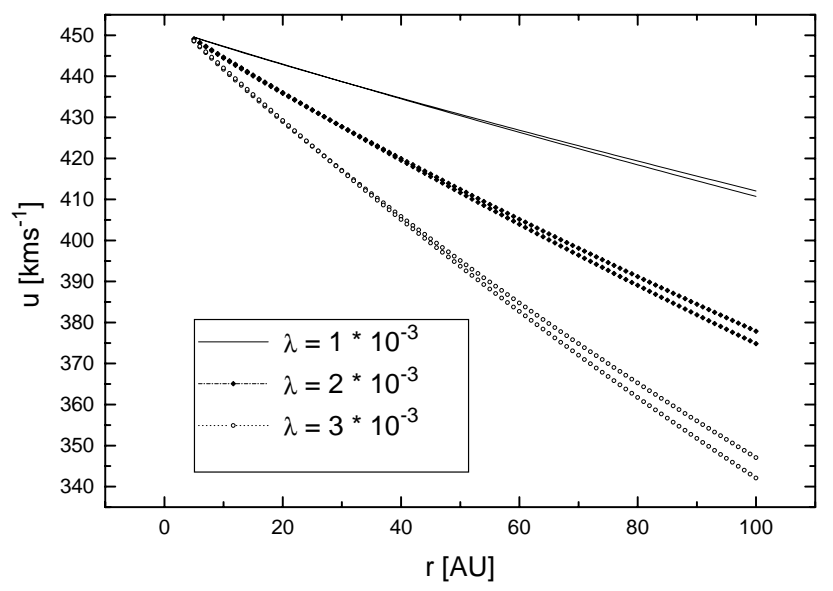

Fig. 2. Shown is the solar wind velocity profile resulting for differerent values of the parameter $\Lambda$ and $r=30$. In all cases the associated dashed curve gives a comparison to the pressure-less case (i.e. $\alpha_{\text {pui }}=0$ ).

The solar wind deceleration resulting from the action of both the momentum loading of the solar wind by injected PUI's and by the PUI pressure gradient is then given by a formula derived in the Appendix B of this paper and given by

$$
\begin{aligned}
U(r)= & U\left(r_{0}\right) \exp \left[\int _ { x = 5 } ^ { x = r / r _ { E } } \frac { d x } { ( 1 + 2 \alpha \xi ) } \left[\frac{\xi}{25 r_{E} \sqrt{x}}+\right.\right. \\
& \left.\frac{3}{2 r_{E}} \frac{\alpha \xi}{x}+\frac{\alpha \Lambda}{5 r_{E}} \xi-\frac{\Lambda}{r_{E}}\left(1+\frac{\alpha}{5}\right)\right]
\end{aligned}
$$

where $\xi$ denotes the local PUI abundance which in the upwind hemisphere for distances $r \geq r_{0}=5 \mathrm{AU}$ is roughly given by

$\xi=1-\exp \left[-\Lambda\left(\frac{r}{r_{0}}-1\right)\right]$

with $\Lambda=\sigma_{e x} n_{H \infty} r_{E}$. In Fig. 2 we have shown solar wind velocity profiles in upwind direction for differerent values of the parameters $\Gamma$ and $\Lambda$.

\section{Conclusions}

We have shown that pick-up ions appearing as ionized $\mathrm{H}$ atoms in the inner heliosphere and freshly injected into the supersonic solar wind experience magnetic cooling, rather than adiabatic cooling, after their injection into the solar wind bulk plasma. This newly derived magnetic cooling is due to the fact that ions, embedded as subsonic ions in a supersonically expanding wind, do not react adiabatically and isentropically, but at their propagation to larger distances co-moving with the bulk of the solar wind plasma they are subject only to energy-conserving pitchangle scattering and magnetic moment conservation processes. As a consequence of that they undergo magnetically induced drifts in velocity space, thus filling the gap between the injection velocity and the thermal energies of solar wind protons. The resulting pick-up ion distribution function is a power law with a power index of $\gamma_{m}=-5$, instead of a power index $\gamma_{a d}=-3 / 2$ found for adiabatic cooling.

Including in addition stochastic pick-up ion acceleration by nonlinear interactions with magnetoacoustic turbulences at quasi-equilibrium saturation levels (see Fisk and Gloeckler, 2007) this then leads to an unbroken power law for pickup ions with a unique power index of $\gamma=-5$ valid from the lowest to the highest velocity resonance borders, i.e. from 0.5 to $100 \mathrm{KeV}$. We fix the absolute spectral intensity of the actually resulting pick-up ion power distribution by the use of its lowest velocity moments like pick-up ion density and pressure and then can calculate the pick-up ion pressure. We can show here that the ratio $\alpha_{\text {pui }}=P_{\text {pui }} /\left[m n_{\text {pui }} U^{2}\right] \simeq 0.3$ at distances $r \geq r_{0}=5 \mathrm{AU}$ behaves as a mildly variable quantity which also characterizes the pick-up ion fluid as a quasiisothermal fluid with a temperature $T_{\text {pui }} \simeq 0.204 \cdot m U^{2} / K$.

As we can show in this paper, the bulk solar wind velocity is decelerated towards larger solar distances with respect to its inner asymptotic value at $5 \mathrm{AU}$. This deceleration clearly is shown in Fig. 2 and clearly is more efficient with increasing interstellar H-atom density $n_{H, \infty}$. Though this deceleration is mainly due to momentum-loading of the solar wind by PUI's, there is in addition an accelerative effect exerted on to the solar wind bulk due to the action of the PUI-pressure gradient. As we can also show in Fig. 2, the PUI pressure by its gradient acts counteractive to the momentum-loading and partly compensates for the effect of momentum loading. In the theoretical approach derived for the PUI pressure in this paper it, however, turns out that the effect of this pressure gradient is not as pronounced as expected from earlier theoretical approaches (see Fahr and Fichtner, 1995; Fahr and Rucinski, 1999) in which the ratio $\alpha_{\text {pui }}$ of PUI pressure and PUI kinetic energy density was found as a constant with a value of $\alpha_{\text {pui }}=0.3$.

\section{Appendix A}

\section{PUI distribution function}

In the following we will confirm by introduction of the solution given by Eq. (10) into the BVE given by Eq. (2) valid in the WF that Eq. (6) actually is the correct solution. Introducing Eq. (10) into Eq. (2) we find:

$$
\begin{aligned}
\frac{\partial}{\partial t}\left(\frac{r \beta\left(\frac{v}{U} r\right)}{U} v^{-3}\right) & +\frac{1}{v^{2}} \frac{\partial}{\partial v}\left(v^{2} \dot{v}_{a d} \frac{r \beta\left(\frac{v}{U} r\right)}{U} v^{-3}\right) \\
& =\beta(r) \frac{1}{2 v^{2}} \delta(v-U)
\end{aligned}
$$


which leads to

$$
\begin{aligned}
v^{-3} \frac{1}{U} \frac{\partial}{\partial t}\left(r \beta\left(\frac{v}{U} r\right)\right) & +\frac{1}{v^{2}} \frac{\partial}{\partial v}\left(v^{2}\left(-U \frac{v}{r}\right) \frac{r \beta\left(\frac{v}{U} r\right)}{U} v^{-3}\right) \\
& =\beta(r) \frac{1}{2 v^{2}} \delta(v-U)
\end{aligned}
$$

and

$$
\begin{aligned}
v^{-1} \frac{1}{U} \frac{\partial}{\partial t}\left(r \beta\left(\frac{v}{U} r\right)\right) & -\frac{\partial}{\partial v}\left(v^{3} \beta\left(\frac{v}{U} r\right) v^{-3}\right) \\
& =\beta(r) \frac{1}{2} \delta(v-U)
\end{aligned}
$$

This furthermore is leading to

$$
\begin{aligned}
v^{-1} \frac{1}{U}\left(U \beta\left(\frac{v}{U} r\right)+r \frac{\partial \beta}{\partial r_{v}} \frac{\partial r_{v}}{\partial t}\right) & -\frac{\partial}{\partial v}\left(\beta\left(\frac{v}{U} r\right)\right) \\
& =\beta(r) \frac{1}{2} \delta(v-U)
\end{aligned}
$$

and

$v^{-1}\left(\beta\left(\frac{v}{U} r\right)+r \frac{\partial \beta}{\partial r_{v}} \frac{v}{U}\right)-\frac{\partial \beta}{\partial r_{v}} \frac{r}{U}=\beta(r) \frac{1}{2} \delta(v-U)$

which finally yields

$v^{-1} \beta\left(\frac{v}{U} r\right)+\frac{\partial \beta}{\partial r_{v}} \frac{r}{U}-\frac{\partial \beta}{\partial r_{v}} \frac{r}{U}=\beta(r) \frac{1}{2} \delta(v-U)$

The above relation then reduces to:

$\beta\left(\frac{v}{U} r\right)=\beta(r) \frac{1}{2} v \delta(v-U)$

and evidently can be rearranged into the following from

$$
1=\beta(r) \cdot \frac{1}{2} \frac{1}{\beta\left(\frac{v}{U} r\right)} v \delta(v-U)
$$

This above equation can then be integrated over $v$

$$
\int_{0}^{U} d v=\beta(r) \cdot \int_{U-\epsilon}^{U+\epsilon} \frac{1}{\beta\left(\frac{v}{U} r\right)} v \delta(v-U) d v
$$

and then evaluates to

$$
U=\beta(r) \cdot \int_{U-\epsilon}^{U+\epsilon} \frac{v}{\beta\left(\frac{v}{U} r\right)} \delta(v-U) d v=U \frac{\beta(r)}{\beta(r)} !
$$

This demonstrates that the PUI distribution function given in Eq. (6) in fact fulfills the Boltzmann-Vlasow equation given by Eq. (2) and thus is the solution of the problem.

\section{Appendix B}

\section{Equation of motion of PUI-modulated winds}

In the following we derive the equation of motion for the PUI-modulated solar wind. We start out with the following equation containing the effect of the PUI-pressure gradient and the momentum loading due to pick-up of new ions by the solar wind:

$\rho U \frac{d U}{d r}=-\frac{d}{d r}\left(\alpha \rho_{i} U^{2}\right)-\left(m_{p} U\right)\left(\sigma_{e x} n_{H} n U\right)$

Carrying out the derivatives then leads to

$$
\begin{aligned}
U \frac{d U}{d r}=-\frac{1}{\rho}\left[\rho_{i} U^{2} \frac{d \alpha}{d r}\right. & +\alpha U^{2} \frac{d \rho_{i}}{d r} \\
& \left.+2 \alpha \rho_{i} U \frac{d U}{d r}\right]-\sigma_{e x} n_{H} U^{2}
\end{aligned}
$$

and furtheron to

$$
\frac{1}{U} \frac{d U}{d r}(1+2 \alpha \xi)=-\left[\xi \frac{d \alpha}{d r}+\alpha \frac{1}{\rho} \frac{d \rho_{i}}{d r}\right]-\frac{\Lambda}{r_{E}}
$$

or

$$
\frac{d \ln U}{d r}=\frac{1}{(1+2 \alpha \xi)}\left(-\xi \frac{d \alpha}{d r}-\alpha \frac{1}{\rho} \frac{d \xi \rho}{d r}-\frac{\Lambda}{r_{E}}\right)
$$

With the explicit derivative of $\alpha_{\text {pui }}(r)$ given in Eq. (53) and $x=r / r_{E}$ one finds

$$
\begin{aligned}
\frac{d \alpha}{d r} & =\frac{d}{d r}\left(\frac{1}{25} \ln \left(\frac{5 \Gamma}{x}\right) \sqrt{x}\right) \\
& =\frac{1}{25 r_{E} x}\left[-\sqrt{x}+\frac{1}{2} \ln \left(\frac{5 \Gamma}{x}\right) \sqrt{x}\right] \\
& =-\frac{1}{25 r_{E} \sqrt{x}}+\frac{1}{2 r_{E}} \frac{\alpha}{x}
\end{aligned}
$$

and obtains

$$
\begin{aligned}
\frac{d \ln U}{d r}=\frac{1}{(1+2 \alpha \xi)}\left(\frac{\xi}{25 r_{E} \sqrt{x}}\right. & -\frac{\xi}{2 r_{E}} \frac{\alpha}{x} \\
& \left.-\alpha \frac{1}{\rho} \frac{d \xi \rho}{d r}-\frac{\Lambda}{r_{E}}\right)
\end{aligned}
$$

Now with the derivative of $\xi(r)$ given in Eq. (60)

$\frac{1}{\rho} \frac{d \xi \rho}{d r}=\frac{d \xi}{d r}+\xi \frac{d \ln \rho}{d r}=-\frac{\Lambda}{5 r_{E}}(\xi-1)+\xi\left(-\frac{2}{r}\right)$

one furthermore finds

$$
\begin{aligned}
\frac{d \ln U}{d r} & =\frac{1}{(1+2 \alpha \xi)}\left(\frac{\xi}{25 r_{E} \sqrt{x}}-\frac{\xi}{2 r_{E}} \frac{\alpha}{x}\right. \\
& \left.-\frac{\alpha \Lambda}{5 r_{E}}(1-\xi)+\alpha \xi\left(\frac{2}{r_{E} x}\right)-\frac{\Lambda}{r_{E}}\right)
\end{aligned}
$$

or finally after integration:

$$
\begin{aligned}
U(r) & =U\left(r_{0}\right) \exp \left[\int _ { 5 } ^ { x = r / r _ { E } } \frac { d x } { ( 1 + 2 \alpha \xi ) } \left[\frac{\xi}{25 r_{E} \sqrt{x}}\right.\right. \\
& \left.\left.+\frac{3}{2 r_{E}} \frac{\alpha \xi}{x}+\frac{\alpha \Lambda}{5 r_{E}} \xi-\frac{\Lambda}{r_{E}}\left(1+\frac{\alpha}{5}\right)\right]\right]
\end{aligned}
$$

Topical Editor B. Forsyth thanks K. Scherer and another anonymous referee for their help in evaluating this paper. 


\section{References}

Bogdan, T. J., Lee, M. A., and Schneider, P.: Coupled quaislinear wave-damping and stochastic acceleration of pick-up ions in the solar wind, J. Geophys. Res., 96, 161-172, 1991.

Chalov, S. V. and Fahr, H. J.: Reflection of preaccelerated pick-up ions at the solar wind termination shock: The seed for anomalous cosmci rays, Sol. Phys., 168, 389-411, 1996.

Chalov, S. V. and Fahr, H. J.: Interplanetary pick-up ion acceleration, Astrophys.Space Sci., 264, 509-525, 1999.

Chalov, S. V. and Fahr, H. J.: Pick-up ion acceleration at the termination shock and the post-shock pick-up ion distribution, Astron. Astrophys., 360, 381-390, 2000.

Chalov, S. V., Alexashov, D. B, and Fahr, H.-J., Reabsorption of self-generated turbulent energy by pick-up protons in the outer heliosphere, Astron. Astrophys., 416, L31-L34, 2004.

Chalov, S. V., Alexashov, D. B., and Fahr, H.-J.: Interstellar pick-up protons and solar wind heating in the outer heliosphere, Astron. Lett., 32, 206-213, 2006a.

Chalov, S. V., Alexashov, D. B., and Fahr, H.-J.: Heating of the solar wind in the outer heliosphere, Astrophys. Space Sci. Trans., 2, 19-25, 2006b.

Chashei, I. V., Fahr, H. J., and Lay, G.: Heating of the solar wind ion species by wave energy dissipation, Adv. Space Res., 32(4), 507-512, 2003.

Chashei, I. V., Fahr, H. J., and Lay, G.: Ion relaxation processes in the heliospheric interface: how perturbed are ion distribution functions?, Adv. Space Res., 35, 2078-2083, 2005.

Fahr, H. J.: The interplanetary hydrogen cone and its solar cycle variations , Astron. Astrophys., 14, 263-271, 1971.

Fahr, H. J.: Global energy transfer from pick-up ions to solar wind protons, Sol. Phys., 208, 335-344, 2002.

Fahr, H. J.: Solar wind heating by an embedded quasi-isothermal pick-up ion fluid, Ann. Geophys., 20, 1509-1518, 2002, http://www.ann-geophys.net/20/1509/2002/.

Fahr, H. J. and Shizgal, B.: Modern exospheric theories and their observational relevance, Rev. Geophys. Space Phys., 21, 75-124, 1983.

Fahr, H.J. and Fichtner, H.: The influence of pick-up ion induced wave pressures on the dynamics of the mass loaded solar wind, Solar Physics, 158, 353-363, 1995.

Fahr, H. J. and Rucinski, D.: Neutral interstellar gas atoms reducing the solar wind Mach number and fractionally neutralizing the solar wind, Astron. Astrophys., 350, 1071-1078, 1999.

Fahr, H. J. and Lay, G.: Remote diagnostic of the heliospheric termination shock using neutralized post-shock pick-up ions as messengers, Astron. Astrophys., 356, 327-334, 2000.

Fahr, H. J. and Chashei, I. V.: On the thermodynamics of MHD wave-heated solar wind protons, Astron. Astrophys., 395, 9911000, 2002.

Fahr, H. J. and Scherer, K.: Perturbations of the solar wind flow by radial and latitudinal pick-up ion pressure gradients, Ann. Geophys., 22, 2229-2238, 2004, http://www.ann-geophys.net/22/2229/2004/.

Fisk, L. A.: The acceleration of energetic particles in the interplanetary medium by transit time damping, J. Geophys. Res., 81, 4633-4640, 1976a.

Fisk, L. A.: On the acceleration of energetic particles in the interplanetary medium, J. Geophys. Res., 81, 4641-4645, 1976 b.

Fisk, L. A., Gloeckler, G., Zurbuchen, T. H., and Schwadron, N.
A.: Ubiquitous statistical acceleration in the solar wind, in: ACE Symposium 2000, edited by: Mewald, R. A., American Institute of Physics, Proceed. ACE-Symposium, edited by: Mewald, D., et al., 221-233, 2000.

Fisk, L. A. and Gloeckler, G.: The common spectrum for accelerated ions in the quiet-time solar wind, Astrophys. J., 640, L79L82, 2006.

Fisk, L. A. and Gloeckler, G.: Thermodynamic constraints on stochastic acceleration in compressional turbulence, Proc. Nat. Acad. Sci., 104(3), 5749-5754, 2007.

Geiss, J., Gloeckler, G., and Mall, U.: Origin of $\mathrm{O}^{+}$pick-up ions in the heliosphere, Astron. Astrophys., 289, 933-938, 1994.

Gloeckler, G., Geiss, J., Balsiger, H., Fisk, L. A., Galvin, A. B. Ipavich, A. B., Ogilvie, K. W., von Steiger, R., and Wilken, B.: Detection of interstellar pick-up hydrogen in the solar system, Science, 261, 70-73, 1993.

Gleeson, L. J. and Axford, W. I.: Cosmic rays in the interstellar medium, Astrophys. J., 149, L115-L118, 1967.

Griffel, D. H. and Davis, L.: The anisotropy of the solar wind, Planet. Space. Sci., 17, 1009-1013, 1969.

Isenberg, P. A.: J. Geophys. Res., 92, 1067-1076, 1987.

Jokipii, J. R.: Propagation of cosmic rays in the solar wind, Rev. Geophys. Space Phys., 9, 27-87, 1971.

Lee, M. A: Coupled hydromagnetic wave excitation and ion acceleration upstream of the earth's bow shock, J.Geophys.Res., 87, 5063-5071, 1982.

Lemaire, J. and Scherer, M.: Kinetic models of the solar wind, J. Geophys. Res., 76, 7479-7490, 1971.

Mall, U.: Pick-up ions in the solar wind, in: The Outer Heliosphere: Beyond the Planets, Copernicus Society, edited by: Scherer, K., Fichtner, H. and et al., 156-163, 2000.

Marsch, E., Mühlhäuser, K. H., Schwenn, R., Rosenbauer,H., Pilipp, W., and Neubauer, F. M.: Solar wind protons: Threediemnsional velcoity distributions and derived plasma parameters measured between 0.3 to $1.0 \mathrm{AU}, \mathrm{J}$. Geophys. Res., 87, 52$59,1982$.

Marsch, E. and Livi, S.: Coulomb self-collision frequencies for non-thermal velocity distributions in the solar wind, Ann. Geophys., 3, 545-556, 1985, http://www.ann-geophys.net/3/545/1985/.

Moebius, E., Rucinski, D., Isenberg, P. A., and Lee, M. A.: Determination of interstellar pickup ion distributions in the solar wind with SOHO and Cluster, Ann. Geophys., 14, 492-502, 1996, http://www.ann-geophys.net/14/492/1996/.

Moebius, E., Rucinski, D., Lee, M. A., and Isenberg, P. A.: Decreases in the antisunward flux of interstellar pick-up $\mathrm{He}^{+}$associated with the radial interplanetary magnetic fields, J. Geophys. Res., 103, 257-263, 1998.

Parker, E. N.: Planet. Space Sci., 13, 9 pp., 1965.

Siewert, M. and Fahr, H. J.: A Boltzmann-kinetical description of an MHD shock with arbitrary field inclinations, Astron. Astrophys., accepted, 2007.

Smith, C. V., Matthaeus, W. H., Zank, G. P., Ness, N. F., Oughton S., and Richardson, J.: Heating of the low latitude solar wind by dissipation of turbulent magnetic fluctuations, J. Geophys. Res., 106, 8523-8531, 2001.

Toptygin, I. N.: in: Cosmic rays in interplanetary magnetic fields, Geophysics and Astrophysics Monographs, D. Reidel Publishing Company, Dordrecht (The Netherlands), 200-220, 1985. 
Vasyliunas, V. M. and Siscoe, G. L.: On the flux and the energy spectrum interstellarious in the solar system, J. Geophys. Res., 81, 1247-1253, 1976. 